\title{
El profesional de enfermería, por la gestión del riesgo, hacia la seguridad del paciente
}

\section{Elena Gómiz León}

Subdirectora de Enfermería. Hospital Severo Ochoa. M adrid

\section{Sra. Directora:}

La seguridad del paciente se considera una prioridad en la asistencia sanitaria, actividad cada vez más compleja, que entraña riesgos potenciales y en la que no existe un sistema capaz de garantizar la ausencia de efectos adversos, ya que se trata de una actividad en la que se combinan factores inherentes al sistema con actuaciones humanas.

La gestión de riesgos es una metodología que se utiliza cada vez más en los servicios sanitarios. Sus principales objetivos son, incrementar la calidad de la asistencia, mejorar la seguridad de los pacientes y de los profesionales sanitarios que la reciben y la llevan a cabo y disminuir los costes evitables.

La preocupación por la calidad de la asistencia, por la seguridad de los pacientes y por la confidencialidad de la información clínica es general en los sistemas sanitarios y se recoge en diferentes normas legales y reglamentarias, con independencia de su modo organizativo y financiero.

E $\mathrm{n}$ todo el mundo occidental y también en nuestro país, las reclamaciones y demandas por una posible deficiencia asistencial sanitaria, muestran un incremento discreto, pero constante. $L$ a gestión de riesgos sanitarios, es una de las vías que se están poniendo

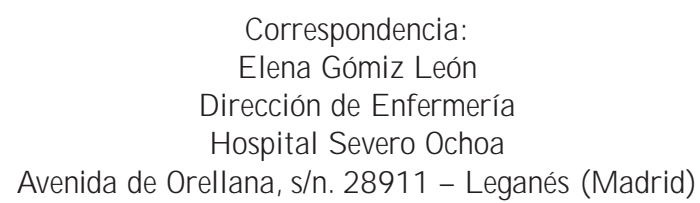

en práctica con el fin de moderar la siniestralidad y su coste en el sector sanitario.

La gestión de riesgos clínicos se basa en la identificación, el análisis y la corrección de las causas que originan daño secundario al paciente tras el tratamiento médico o la administración de cuidados. Es imposible evitar completamente el daño al paciente, toda acción humana conlleva un margen de error; sin embargo es posible identificar, estudiar, controlar y minimizar ese daño; en definitiva, gestionar el riesgo que pueda sufrir el paciente.

La Consejería de Sanidad y Consumo ha creado el observatorio de Riesgos Sanitarios de la Comunidad de Madrid, orientada a incrementar la calidad de la asistencia y la seguridad de los profesionales y pacientes en el entorno sanitario.

No se nos escapa, que en el mundo de la Nefrología, la tecnología avanza cada vez más deprisa, y los profesionales que nos dedicamos a ella, sabemos que la seguridad de los equipos cada vez es mayor, los tratamientos más complejos, que el perfil del paciente ha cambiado, demanda información sobre su tratamiento, efectos y quiere ser participe en todo momento de lo que le acontece, nosotros sabemos que también tenemos que darle esa información, (Ley de A utonomía del paciente, derechos y deberes) y que la Comunicación, algo tan simple y complicado a la vez, va tomando más y mayor importancia.

Hay que recordar que los problemas de seguridad se producen como consecuencia de múltiples causas, no solo el fallo humano es la principal explicación. Los expertos proponen un enfoque sistémico, recordando 
que la aparición de los mismos depende, en gran medida, de múltiples factores contribuyentes relacionados con el sistema. Es frecuente encontrar después del análisis en profundidad de un problema de seguridad, causas relacionadas con la comunicación, la ergonomía, la fatiga, la dotación del personal, la supervisión o la formación inadecuadas.

Por tanto, muchos de los eventos adversos se deben a situaciones latentes del propio sistema y no a negligencias 0 imprudencias del personal.

Son muchas las líneas de actuación que deben de ponerse en marcha para la mejora de la seguridad de los pacientes y alcanzar algunos de los objetivos formulados. Algunas de las recomendaciones siguientes se desprenden del análisis de los informes internacionales:

- Establecer un enfoque consistente para conseguir liderazgo, investigación, herramientas y protocolos para potenciar el conocimiento sobre la seguridad de los pacientes.

- Identificar los problemas de seguridad y aprender de ellos mediante sistemas de registro y notificación de carácter obligatorio o voluntario.

- Formular estándares de mejora de la seguridad por parte de gobiernos, sociedades científicas y profesionales.

- Implementar prácticas seguras en los niveles de la prestación asistencial.

El profesional sanitario es el ejecutor de la acción asistencial, controlador de los procesos y responsable de los resultados, y es evidente que su responsabilidad, la buena práctica (formación, experiencia y trabajo en equipo) y las herramientas de gestión clínica son los factores que más pueden influir en la minorización del riesgo.

No se nos puede olvidar, que el usuario no recibe toda la información sobre el proceso, SU PROCESO, además las expectativas de los pacientes cambian y el profesional no lo sabe ¿conocemos las verdaderas necesidades de los pacientes?, ¿ sabemos expresarles el contenido de nuestro trabajo?

La mejora de la comunicación es reto actual, para muchos de los que nos dedicamos a la gestión sanitaria, mejorar la comunicación interna en todos los niveles, de forma bidireccional, facilitando la disponibilidad de los recursos posibles, favorece la disminución de fallos o su detección precoz.

Desde nuestra Sociedad, debemos impulsar y difundir la cultura de la gestión del riesgo, avanzando, no solo en tecnología, también en formación, en diferentes temas, necesarios para alcanzar la excelencia de la que goza la E nfermería Nefrológica.

\section{Bibliografía}

1. Aranaz J M, Aibar C, Gea MT, León MT. Los efectos adversos en la asistencia hospitalaria. Una revisión crítica. Med Clin (Barc) 2004; 123:21-5.

2. Aibar C, Aranaz J M . ¿Pueden evitarse los sucesos adversos relacionados con la atención hospitalaria? Anales del Sistema Sanitario de Navarra. 2003; 26:195-209 (citado 4 Nov 2004). Disponible en: http:/www.cfnavarra.es/salud/anales/textos/vol26/n2/colaba.html.

3. Mira J . ¿Deben compartir los riesgos de la toma de decisiones los pacientes? E n: A ranaz J M, Vitaller J, y Grupo de Estudio del Proyecto IDEA: Identificación de E fectos Adversos, editores. De las complicaciones y efectos adversos a la gestión de los riesgos de la asistencia sanitaria. E studios para la salud, n-13. Valencia: Generalitat Valenciana 2004.

4. Sistemas de registro y notificación de incidentes y eventos adversos. Secretaría General de Sanidad. Agencia de calidad del Sistema Nacional de Salud. Ministerio de Sanidad y Consumo. 